\title{
Correction for: Integrative analysis of exosomal microRNA-149-5p in lung adenocarcinoma
}

\author{
Wen Tian' ${ }^{1}$, He Yang ${ }^{1}$, Baosen Zhou ${ }^{1,2}$ \\ ${ }^{1}$ Department of Clinical Epidemiology, First Affiliated Hospital, China Medical University, Shenyang, China \\ ${ }^{2}$ Department of Epidemiology, School of Public Health, China Medical University, Shenyang, China
}

Correspondence to: Baosen Zhou; email: $\underline{\text { bszhou@cmu.edu.cn }}$

Original article: Aging (Albany NY) 2021; 13: pp 7382-7396

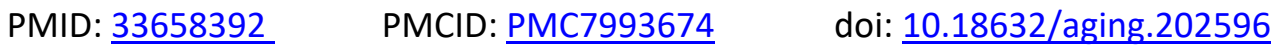

This article has been corrected: The authors requested replacement of panel 6B, in which the left panel (normal tissue stained for BCL2L2) was an accidental duplication of the left panel of Figure 6A (normal tissue stained for AMOTL2). All of the results in Figure 6 were extracted from THPA online database (https://www.proteinatlas.org/). The corrected web link for the left panel of Figure 6B is https://www.proteinatlas.org/ENSG00000129473-BCL2L2/tissue/lung\#img. This change does not affect the results or conclusions of this work.

New Figure 6 is presented below. 
A

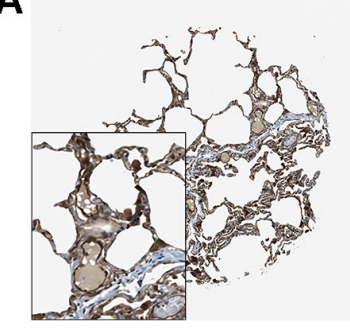

C

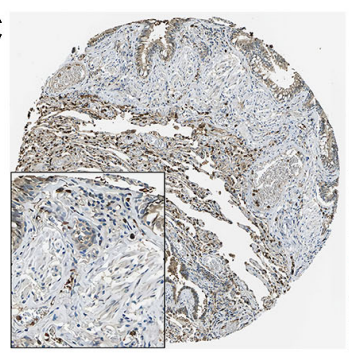

E

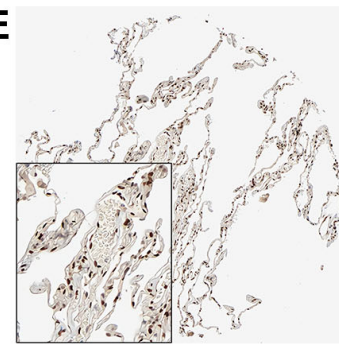

G

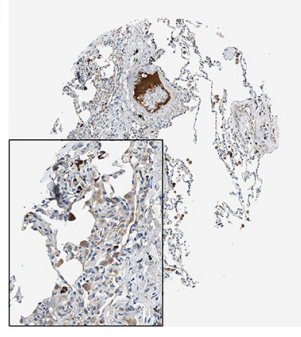

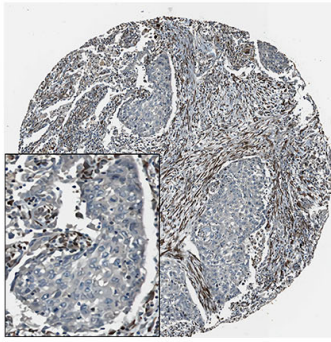

B
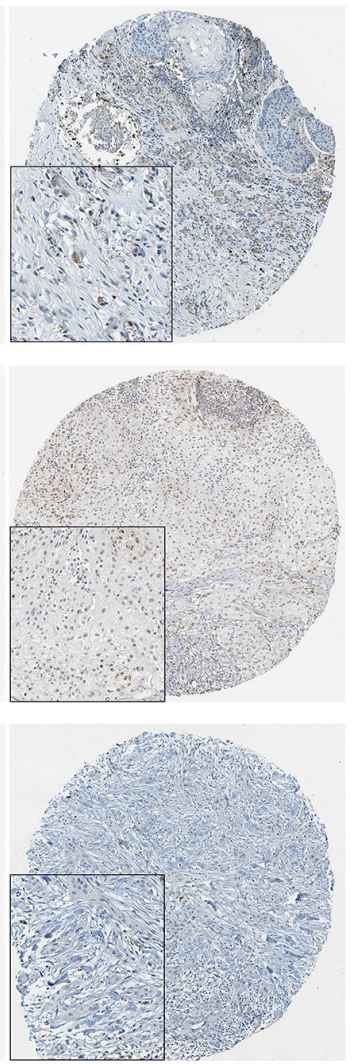

D

$\mathbf{F}$

H
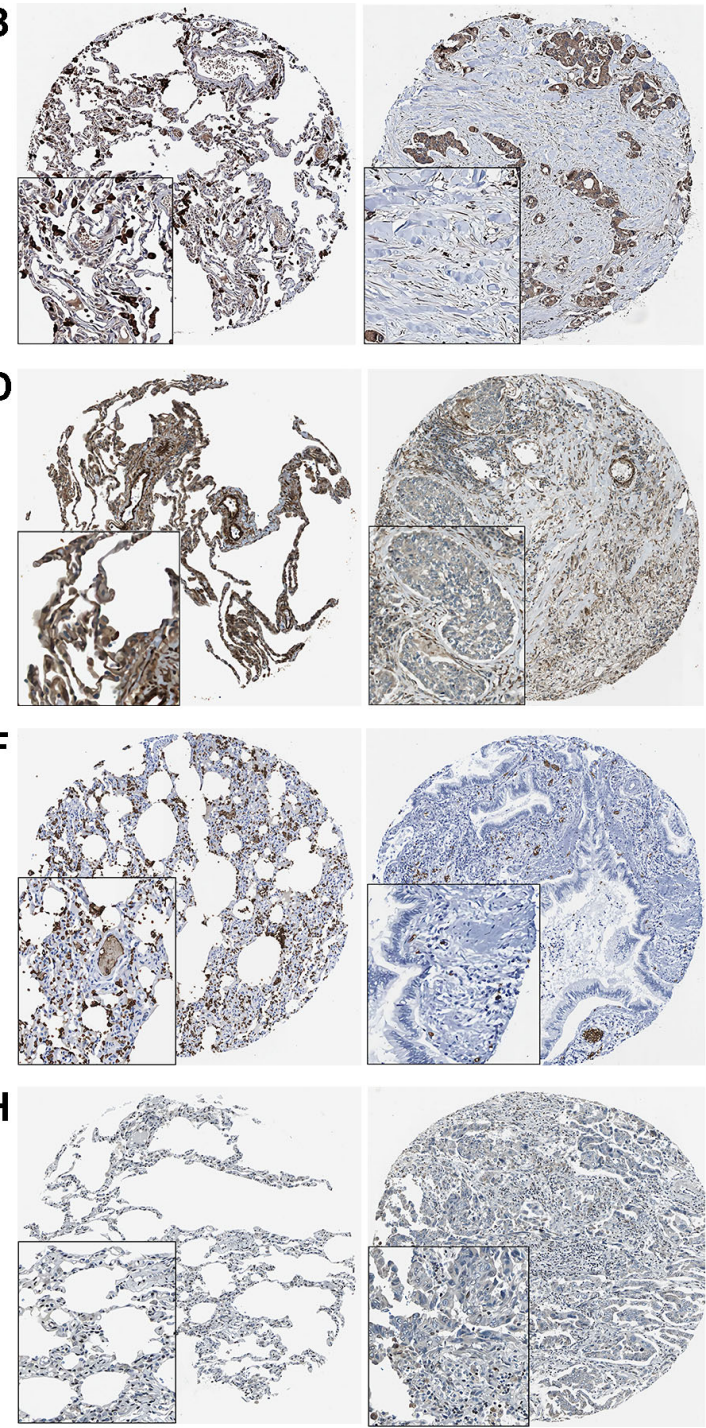

Figure 6. The THPA results of 8 target genes in normal and tumor tissues. (A) AMOTL2. (B) BCL2L2. (C) CACDH1. (D) MSRB3. (E) NFIB. (F) S1PR2. (G) SORT1. (H) SRF. 